\title{
Availability of Selected (Pollutant) Elements and their Influence on Soil Composition in Urban Area
}

\author{
Michaela Zeiner, ${ }^{\mathrm{a}, *}$ Iva Juranović Cindrić, ${ }^{\mathrm{b}}$ Marina Ivanović, ${ }^{\mathrm{c}}$ Gordana Medunić, ${ }^{\mathrm{d}}$ Štefica Kampić, ${ }^{\mathrm{d}}$ \\ Nenad Tomašic, ${ }^{d}$ and Gerhard Stingeder ${ }^{\mathrm{a}}$ \\ ${ }^{a}$ Division of Analytical Chemistry, Department of Chemistry, BOKU - University of Natural Resources and Life Sciences, \\ Muthgasse 18, 1190 Vienna, Austria \\ ${ }^{\mathrm{b}}$ Laboratory of Analytical Chemistry, Faculty of Science, University of Zagreb, Horvatovac 102a, 10000 Zagreb, Croatia \\ ${ }^{\mathrm{c}}$ Institute of Mineralogy and Petrology, Department of Geology, Faculty of Science, \\ University of Zagreb, Horvatovac 95, 10000 Zagreb, Croatia
}

RECEIVED NOVEMBER 21, 2013; REVISED FEBRUARY 14, 2014; ACCEPTED JULY 7, 2014

\begin{abstract}
Interest in growing fruits and vegetables in private gardens is rising due to nowadays ecological awareness. Avoiding artificial fertilizers and plant protection products does not guarantee the absence of toxic substances, especially heavy metals in the soil and thus in the fruits harvested. Due to either geological bedrock weathering or environmental pollution, garden soils may be rich in certain potentially toxic elements. In the present study ten garden soils from central Croatia have been analysed by the BCR method for the contents and bioavailability of aluminium, cadmium, chromium, cobalt, copper, lead, manganese, nickel and zinc.

The total amounts of the elements are in the concentration range as reported for agricultural soils in different geographical regions. Only two soils of the capital Zagreb have higher concentrations of pollutant metals, such as chromium, cobalt, copper, lead, manganese and zinc. Regarding nutrients, all soils have met the needs of common garden plants.
\end{abstract}

Keywords: BCR; ICP-AES; heavy metals; plant nutrients; garden soils; pollutants

\section{INTRODUCTION}

The importance of home gardening is rising in the last years due to the elevated ecological awareness. Fertilizers and plant protection products are considered to lower food quality by their specific toxic properties. Organic farming represents growing plants without synthetic fertilizers and pesticides or genetically modified organisms; however, the inorganic composition of the soils is not regulated. ${ }^{1}$ Only general limits of levels in soils used for agricultural purposes are given by different jurisdictions. ${ }^{2,3}$ In general, plants are useful indicators of environmental heavy metal contamination and can be used to monitor pollution across both spatial and temporal scales. ${ }^{4}$ Fruits produced by organic agriculture in private gardens may also contain toxic elements, such as cadmium, lead, and arsenic due to contamination via air or by uptake from polluted soils.

The favorite products of home gardening are vegetables, like tomatoes and lettuce, and fruits, especially many types of berries, e.g. strawberries, blackberries, raspberries and currants.

Each of these plants has special requirements regarding micro-nutrients and trace elements, i.e. vitamins or minerals needed in small amounts for normal function of the organism. That a micro-nutrient is essential is indicated by its presence in healthy tissue and that its uptake and distribution are homeostatic control. Only small amounts of such nutrients are needed and any deficiency can cause diseases or ill-health, retarded growth and poor harvest. Plants require the following elements: nitrogen $(\mathrm{N})$, phosphorus $(\mathrm{P})$, calcium $(\mathrm{Ca})$, magnesium $(\mathrm{Mg})$, manganese $(\mathrm{Mn})$, iron $(\mathrm{Fe})$, boron (B), copper $(\mathrm{Cu})$, zinc $(\mathrm{Zn})$, and aluminium $(\mathrm{Al}){ }^{5}$

Plants tend to selectively uptake and accumulate certain metals as ions; the level of essential elements depends where the plant is grown, i.e. it is affected by the geochemical characteristics of a soil. The resulting elemental pattern is thus determined by the bioavailability of the elements present in soil and the selectivity of their accumulation. In plants, the major uptake occurs

\footnotetext{
* Author to whom correspondence should be addressed. (E-mail: michaela.zeiner@boku.ac.at)
} 


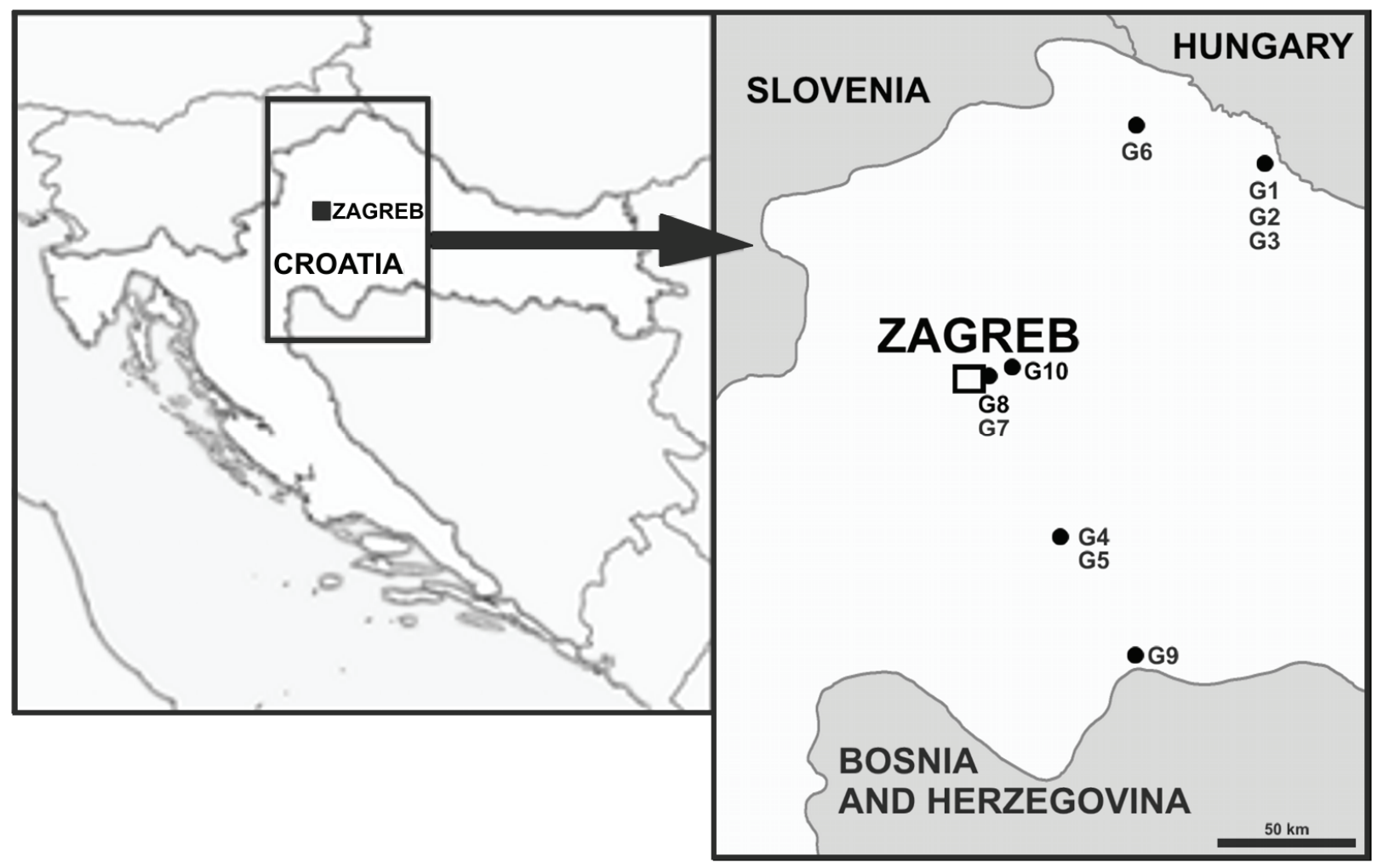

Figure 1. Soil sampling localities.

via their roots. Additional sources of these elements are environmental parameters, such as rainfall, atmospheric dusts (dry deposition), plant protection agents and fertilizers that can be absorbed through their leaves. ${ }^{6}$ Soil analysis is important since the chemical composition of soil reflects both the soil bedrock and the anthropogenic inputs such as pollutants from industrial processes. Information on the mobility or bioavailability of metals however is scant, and special extractions steps are needed to determine the bioavailability of the elements in the plant. ${ }^{7}$ The most frequently applied method is recommended by the European Commission, Community Bureau of Reference (BCR) for sediments and soils, due to its simplicity. ${ }^{8}$

The aim of the present study was the quantitative determination of the elemental content of ten private garden soils from central Croatia. Toxic as well as essential elements were selected, namely $\mathrm{Al}, \mathrm{Cd}, \mathrm{Co}, \mathrm{Cr}$, $\mathrm{Cu}, \mathrm{Fe}, \mathrm{K}, \mathrm{Mg}, \mathrm{Mn}, \mathrm{Ni}, \mathrm{Pb}$, and $\mathrm{Zn}$. Their availability was studied by the BCR method.

\section{MATERIALS AND METHODS}

\section{Soil Samples}

The garden soil samples from the A-horizon were collected in September 2009 at ten sampling sites in Zagreb and its surroundings (see Figure 1). The bedrock geology of the sampling sites is shown in Table 1. After collection the soil samples were dried for 24 hours at $105{ }^{\circ} \mathrm{C}$, ground with a metal-free device and stored at a cool place.

\section{Chemicals and Glassware}

Nitric acid $\left(\mathrm{HNO}_{3} ; 65 \%\right.$ w/w p.a.) and the ICP Multielement Standard IV, both from Merck (Darmstadt, Germany), were used to prepare the standard calibration solutions as well as for the digestion step. The reagents for the extraction steps, i.e. acetic acid $\left(\mathrm{CH}_{3} \mathrm{COOH}\right)$, hydroxylamine hydrochloride $\left(\mathrm{NH}_{2} \mathrm{OH} \times \mathrm{HCl}\right)$, hydrogen peroxide $\left(\mathrm{H}_{2} \mathrm{O}_{2}\right)$, ammonium acetate $\left(\mathrm{NH}_{4} \mathrm{C}_{2} \mathrm{H}_{3} \mathrm{O}_{2}\right)$ and hydrochloric acid $(\mathrm{HCl})$ were analytical grade and purchased from Kemika (Zagreb, Croatia). Before use all glassware was soaked in $7 \mathrm{~mol} / \mathrm{L}$ nitric acid for 24 hours, and then rinsed with supra-pure water.

\section{Extraction Procedure}

Sequential extraction was based on a modified BCR protocol. ${ }^{9}$ The acid-soluble and exchangeable fraction in step 1 was prepared by adding $40 \mathrm{~mL}$ of acetic acid $(0.11 \mathrm{~mol} / \mathrm{L})$ per approx. $1 \mathrm{~g}$ of dry soil sample and shaking for 16 hours at room temperature by an orbital shaker at $250 \mathrm{rpm}$. The residue was treated with $40 \mathrm{~mL}$ of $0.5 \mathrm{~mol} / \mathrm{L}$ hydroxylamine $\mathrm{HCl}(\mathrm{pH}=2)$ to obtain the reducible fraction (step 2). The extraction condi- 
Table 1. Bedrock geology of the sampling area for the investigated soil samples

\begin{tabular}{lll}
\hline Sample & Locality & Bedrock geology \\
\hline G1 & Legrad & Alluvial sediments (gravels, sands and silts) (Holocene) \\
G2 & Legrad & Alluvial sediments (gravels, sands and silts) (Holocene) \\
G3 & Legrad & Alluvial sediments (gravels, sands and silts) (Holocene) \\
G4 & Sisak & Alluvial sediments (pebbles, sands and silts) (Holocene) \\
G5 & Sisak & Alluvial sediments (gravels, sands and silts) (Holocene) \\
G6 & Čakovec & Gravels and sands (Holocene) \\
G7 & Zagreb & Clastitic sediments (Pliocene-Quaternary) \\
G8 & Zagreb & Clastitic sediments (Pliocene-Quaternary) \\
G9 & Kostajnica & Carbonate and clastic sediments (Lower Sarmatian/Panonian) \\
G10 & Zagreb & Carbonate and clastic sediments (Lower Tortonian) \\
\hline
\end{tabular}

tions were as in step 1. Step 3 (the oxidisable fraction) was prepared by adding of $10 \mathrm{~mL}$ of $8.8 \mathrm{~mol} / \mathrm{L}$ hydrogen peroxide and $50 \mathrm{~mL}$ of $1.0 \mathrm{~mol} / \mathrm{L}$ ammonium acetate $(\mathrm{pH}=2)$ to the residue from step 2 and by using again the same extraction conditions as above. Finally, the residue from step 3 was leached with aqua regia. The extracts from each step were separated from the solid by centrifugation (3000 rpm for $20 \mathrm{~min}$ ), decanted into polyethylene bottles and stored at $4{ }^{\circ} \mathrm{C}$ until analysed.

\section{ICP-AES Measurements}

The instrument used was a Prodigy High Dispersive ICP spectrometer operating in simultaneous mode; at settings listed in Table 2. All measurements were carried out in triplicate at the emission lines shown in Table 3.

The calibration standard solutions at concentrations of $0.05,0.10,0.20,0.50,1.0,2.0$, and $5.0 \mathrm{mg} / \mathrm{L}$

Table 2. ICP-AES operating conditions

\begin{tabular}{|c|c|}
\hline Instrument & Prodigy High Dispersive ICP \\
\hline Spectrometer & $\begin{array}{l}\text { High resolution echelle polychromator } \\
\text { Large format programmable array } \\
\text { detector (L-PAD) }\end{array}$ \\
\hline RF-Generator & $40 \mathrm{MHz}$ “free-running” \\
\hline Output power & $1.1 \mathrm{~kW}$ \\
\hline Argon flow & $\begin{array}{l}\text { Coolant: } 18 \mathrm{~L} / \mathrm{min} \\
\text { Auxiliary: } 0.8 \mathrm{~L} / \mathrm{min} \\
\text { Nebulizer: } 1 \mathrm{~L} / \mathrm{min}\end{array}$ \\
\hline Peristaltic pump & $1.0 \mathrm{~mL} / \mathrm{min}$ \\
\hline Nebulizer & Pneumatic (glass concentric) \\
\hline Spray chamber & Glass cyclonic \\
\hline Plasma viewing & Axial \\
\hline $\begin{array}{l}\text { Replicates for } \\
\text { each analysis run }\end{array}$ & 3 \\
\hline Sample uptake delay & $30 \mathrm{~s}$ \\
\hline
\end{tabular}

were prepared in $2 \% \mathrm{w} / \mathrm{w} \mathrm{HNO}_{3}$ from a multi-element standard stock solution (1000 mg/L).

For all analytes and all types of samples the repeatability and precision of the method were determined. The sensitivity of the method was estimated from the slope of the calibration curve. The accuracy was estimated from the recovery of the analytes from the extraction solutions spiked at 0.5 and $2 \mathrm{mg} / \mathrm{L}$. Standard reference materials supplied by the International Atomic Energy Agency (Vienna, Austria) were treated and measured like the samples: IAEA-SL1 (lake sediment), IAEA-Soil 7, IAEA 405 (stream sediments) and IAEA SL 3 (lake sediment).

\section{RESULTS AND DISCUSSION}

\section{ICP-AES Validation}

The accuracy of the method was evaluated by determining the recoveries by the analysis of CRMs and by spiking experiments of all analytes in the extracts of all four extraction steps. The mean recoveries of both concentrations range from $90-112 \%$ and are listed in Table 3 along with their limit of detection (LOD). Regarding the tested CRMs the recoveries ranged from 87 to $110 \%$ for the certified elements. Except for a few elements in single steps all LODs are $<3 \mu \mathrm{g} / \mathrm{L}$. The precision for all elements ranged from 0.5 to $2.4 \%$ in the four extraction steps. The repeatability was $<1.3 \%$, the intermediate precision $<5.7 \%$, the day-to-day reproducibility $<6.8$ $\%$ and the overall uncertainty of measurement was estimated to be $4-8.5 \%$ for all elements analysed. ${ }^{10}$ The validation of analytical procedure optimized was found to be acceptable for environmental analyses. ${ }^{11}$

\section{Metal Content and Extractability}

The results (expressed in $\mu \mathrm{g} / \mathrm{g}$ soil) for each extraction step together with the percentage extracted per step are 
Table 3. Recoveries and limits of detection for the elements in the extract solution of all four sequential extraction steps

\begin{tabular}{|c|c|c|c|c|c|c|c|c|}
\hline \multirow[b]{2}{*}{$\begin{array}{l}\text { Element and } \\
\text { wavelength / nm }\end{array}$} & \multicolumn{2}{|c|}{$\begin{array}{c}\text { step } 1 \\
\left(\mathrm{CH}_{3} \mathrm{COOH}\right) \\
\end{array}$} & \multicolumn{2}{|c|}{$\begin{array}{c}\text { step } 2 \\
\left(\mathrm{NH}_{2} \mathrm{OH} \times \mathrm{HCl}\right)\end{array}$} & \multicolumn{2}{|c|}{$\begin{array}{c}\text { step } 3 \\
\left(\mathrm{H}_{2} \mathrm{O}_{2}+\mathrm{CH}_{3} \mathrm{COONH}_{4}\right)\end{array}$} & \multicolumn{2}{|c|}{$\begin{array}{c}\text { step } 4 \\
\text { (aqua regia) }\end{array}$} \\
\hline & $\frac{\text { Recovery }}{\%}$ & $\frac{\mathrm{LOD}}{\mu \mathrm{g} \mathrm{L^{-1 }}}$ & $\frac{\text { Recovery }}{\%}$ & $\frac{\mathrm{LOD}}{\mu \mathrm{g} \mathrm{L} \mathrm{L}^{-1}}$ & $\frac{\text { Recovery }}{\%}$ & $\frac{\mathrm{LOD}}{\mu \mathrm{g} \mathrm{L^{-1 }}}$ & $\frac{\text { Recovery }}{\%}$ & $\frac{\mathrm{LOD}}{\mu \mathrm{g} \mathrm{L}}$ \\
\hline Al 308.215 & 101 & 2.79 & 97 & 2.26 & 106 & 2.20 & 98 & 2.00 \\
\hline Cd 214.441 & 111 & 0.02 & 103 & 0.15 & 103 & 0.21 & 103 & 0.58 \\
\hline Co 228.615 & 103 & 0.95 & 98 & 2.60 & 100 & 0.76 & 93 & 1.62 \\
\hline Cr 206.149 & 101 & 1.99 & 98 & 1.71 & 111 & 1.68 & 101 & 2.01 \\
\hline $\mathrm{Cu} 224.700$ & 97 & 4.74 & 102 & 0.64 & 100 & 1.97 & 96 & 1.62 \\
\hline Mn 257.610 & 101 & 0.83 & 97 & 5.97 & 97 & 1.71 & 99 & 0.12 \\
\hline Ni 231.604 & 110 & 6.23 & 98 & 0.13 & 112 & 0.97 & 99 & 1.57 \\
\hline $\mathrm{Pb} 220.353$ & 105 & 2.41 & 110 & 1.50 & 105 & 3.17 & 102 & 4.85 \\
\hline Zn 213.856 & 92 & 5.28 & 100 & 3.84 & 99 & 0.18 & 95 & 0.37 \\
\hline
\end{tabular}

listed for all elements investigated in Table $4 \mathrm{a}-1$. Each element is discussed separately. The elemental soil composition (Figure 2) shows the median total amounts of the metals analysed. The extraction behavior of all elements is compared by cluster analysis (Figure 3). Aluminium and chromium (three valent ions) are grouped together in all steps except in step 3. Each extraction step showed a different pattern.

\section{Aluminium}

Aluminium, one of the most important non-essential elements in soils, can affect the development of plant roots and reduce the plant's growth rate. ${ }^{12}$ The total amounts of aluminium ranged from 6.5 to $10.7 \mathrm{mg} / \mathrm{g}$ dry soil, while $73-89 \%$ were not extracted. This can be attributed to the high binding affinity of Al to humic substances. ${ }^{12}$ Only less than $1 \%$ was leached by acetic acid. In step 2, 7-11\% were extracted except from soils G7 and G8 with $14 \%$ and $19 \%$, respectively. In the oxidisable fraction 2.7 up to $12 \%$ were extracted, with a median of $4.7 \%$. Similar extraction behaviour was also described by Li et al., ${ }^{13}$ who analysed soils in mining and smelting areas in England. There the total Al amounts in soil were mainly higher, approx. $20 \mathrm{~g} / \mathrm{kg}$ (range: $7.6-35.4 \mathrm{mg} / \mathrm{g}$ ). No limit concentrations were found for $\mathrm{Al}$ in agricultural soils. ${ }^{14}$

\section{Cadmium}

$\mathrm{Cd}$ in soil occurs either naturally or is derived from human activities. ${ }^{15}$ In the analysed soils cadmium levels

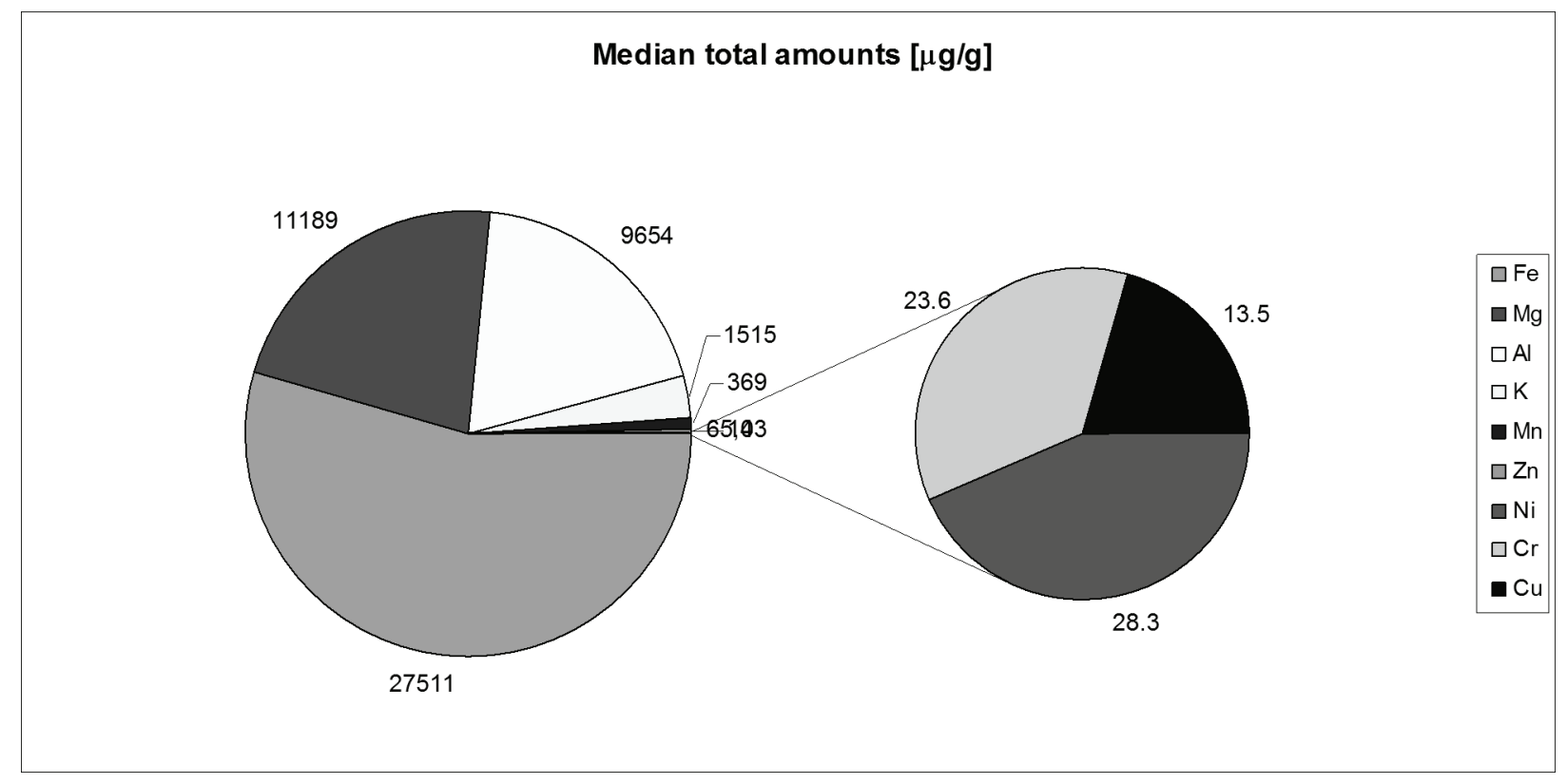

Figure 2. Median total soil concentrations of elements analysed (soil treated with aqua regia). 


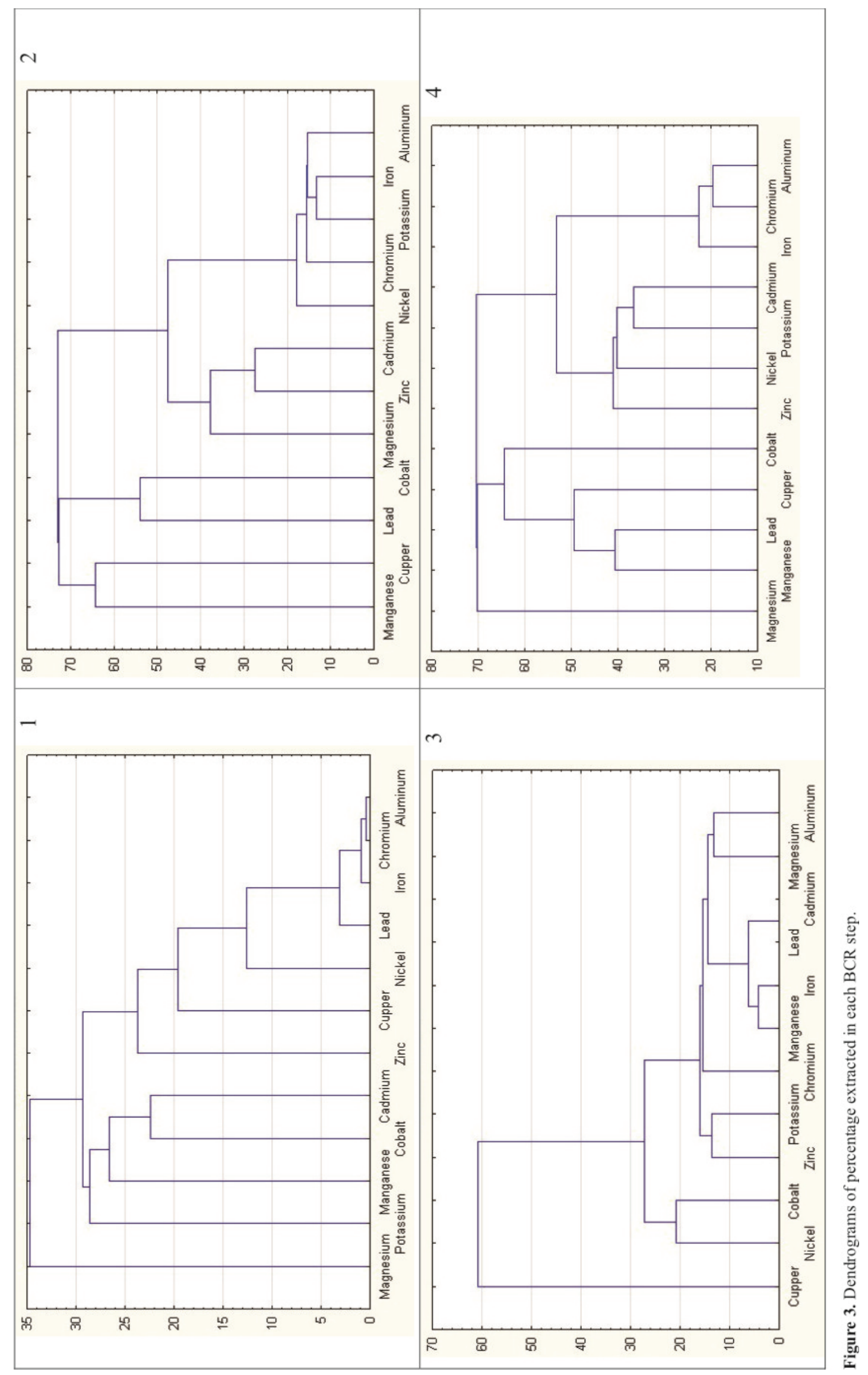

Croat. Chem. Acta 88 (2015) 23. 
Table 4. Amount and percentage of nutrient extracted per step (step $1-\mathrm{CH}_{3} \mathrm{COOH}$; step $2-\mathrm{NH}_{2} \mathrm{OH} \times \mathrm{HCl}$; step $3-\mathrm{H}_{2} \mathrm{O}_{2}+$ $\mathrm{CH}_{3} \mathrm{COONH}_{4}$; step 4 - aqua regia)

\begin{tabular}{lcccccccccc}
\hline a - Aluminium & \multicolumn{1}{c}{$\mu \mathrm{g} / \mathrm{g}$} \\
\cline { 2 - 10 } & step 1 & step 2 & step 3 & step 4 & sum extracted & step 1 & step 2 & step 3 & step 4 & sum extracted \\
\hline G1 & 3.75 & 709 & 481 & 8336 & 9529 & 0.0394 & 7.44 & 5.04 & 87.5 & 100 \\
G2 & 2.74 & 857 & 318 & 6712 & 7889 & 0.0347 & 10.9 & 4.03 & 85.1 & 100 \\
G3 & 2.95 & 912 & 551 & 6336 & 7802 & 0.0378 & 11.7 & 7.07 & 81.2 & 100 \\
G4 & 30.4 & 901 & 430 & 8417 & 9779 & 0.311 & 9.22 & 4.40 & 86.1 & 100 \\
G5 & 1.39 & 565 & 284 & 5702 & 6552 & 0.0213 & 8.62 & 4.34 & 87.0 & 100 \\
G6 & 10.7 & 680 & 217 & 7057 & 7965 & 0.134 & 8.54 & 2.73 & 88.6 & 100 \\
G7 & 33.9 & 1543 & 1276 & 7881 & 10735 & 0.316 & 14.4 & 11.9 & 73.4 & 100 \\
G8 & 42.4 & 1853 & 623 & 7455 & 9973 & 0.425 & 18.6 & 6.20 & 74.8 & 100 \\
G9 & 89.4 & 972 & 1192 & 7609 & 9863 & 0.907 & 9.86 & 12.1 & 77.1 & 100 \\
G10 & 1.19 & 926 & 349 & 9096 & 10372 & 0.0115 & 8.93 & 3.36 & 87.7 & 100 \\
\hline
\end{tabular}

\begin{tabular}{lcccccccccc}
\hline b - Cadmium & \multicolumn{1}{c}{$\mu \mathrm{g} / \mathrm{g}$} \\
\cline { 2 - 11 } & step 1 & step 2 & step 3 & step 4 & sum extracted & step 1 & step 2 & step 3 & step 4 & sum extracted \\
\hline G1 & 0.302 & 0.706 & <LOD & 1.05 & 2.06 & 14.7 & 34.3 & 0 & 51.0 & 100 \\
G2 & 0.339 & 0.491 & <LOD & 0.684 & 1.51 & 22.4 & 32.4 & 0 & 45.2 & 100 \\
G3 & 0.308 & 0.654 & <LOD & 0.639 & 1.60 & 19.2 & 40.9 & 0 & 39.9 & 100 \\
G4 & 0.146 & 0.248 & <LOD & 1.13 & 1.53 & 9.58 & 16.2 & 0 & 74.2 & 100 \\
G5 & 0.349 & 0.489 & <LOD & 0.681 & 1.52 & 23.0 & 32.2 & 0 & 44.9 & 100 \\
G6 & 0.143 & 0.277 & <LOD & 0.926 & 1.35 & 10.6 & 20.6 & 0 & 68.8 & 100 \\
G7 & 0.148 & 0.343 & <LOD & 1.14 & 1.63 & 9.04 & 21.0 & 0 & 69.9 & 100 \\
G8 & 0.196 & 0.413 & <LOD & 1.44 & 2.05 & 9.57 & 20.1 & 0 & 70.3 & 100 \\
G9 & 0.174 & 0.310 & <LOD & 0.765 & 1.25 & 13.9 & 24.8 & 0 & 61.3 & 100 \\
G10 & 0.329 & 0.191 & <LOD & 1.26 & 1.78 & 18.5 & 10.8 & 0 & 70.7 & 100 \\
\hline
\end{tabular}

\begin{tabular}{|c|c|c|c|c|c|c|c|c|c|c|}
\hline \multirow[t]{2}{*}{ c - Chromium } & \multicolumn{5}{|c|}{$\mu \mathrm{g} / \mathrm{g}$} & \multicolumn{5}{|c|}{$\%$} \\
\hline & step 1 & step 2 & step 3 & step 4 & sum extracted & step 1 & step 2 & step 3 & step 4 & sum extracted \\
\hline G1 & $<$ LOD & 0.930 & $<$ LOD & 23.0 & 23.9 & 0 & 3.89 & 0 & 96.1 & 100 \\
\hline $\mathrm{G} 2$ & $<\mathrm{LOD}$ & 1.18 & 0.909 & 18.5 & 20.6 & 0 & 5.75 & 4.42 & 89.8 & 100 \\
\hline G3 & $<\mathrm{LOD}$ & 1.08 & 1.94 & 15.7 & 18.7 & 0 & 5.75 & 10.4 & 84.1 & 100 \\
\hline G4 & 0.0853 & 1.28 & 1.62 & 22.0 & 25.0 & 0.342 & 5.12 & 6.51 & 88.0 & 100 \\
\hline G5 & 0.0592 & 2.05 & 3.06 & 18.1 & 23.2 & 0.255 & 8.83 & 13.2 & 77.7 & 100 \\
\hline G6 & 0.00792 & 0.374 & 0.396 & 9.83 & 10.6 & 0.0747 & 3.53 & 3.73 & 92.7 & 100 \\
\hline G7 & 0.140 & 4.32 & 5.59 & 31.3 & 41.3 & 0.338 & 10.4 & 13.5 & 75.7 & 100 \\
\hline G8 & 0.303 & 10.1 & 10.4 & 42.6 & 63.4 & 0.478 & 15.8 & 16.5 & 67.2 & 100 \\
\hline G9 & 0.124 & 1.00 & 2.79 & 15.9 & 19.9 & 0.625 & 5.04 & 14.1 & 80.3 & 100 \\
\hline G10 & 0.0350 & 0.0507 & 0.606 & 29.0 & 29.6 & 0.118 & 0.171 & 2.04 & 97.7 & 100 \\
\hline \multirow[t]{2}{*}{ d - Cobalt } & \multicolumn{5}{|c|}{$\mu \mathrm{g} / \mathrm{g}$} & \multicolumn{5}{|c|}{$\%$} \\
\hline & step 1 & step 2 & step 3 & step 4 & sum extracted & step 1 & step 2 & step 3 & step 4 & sum extracted \\
\hline G1 & 0.554 & 3.27 & 0.598 & 0.135 & 4.55 & 12.2 & 71.7 & 13.1 & 2.96 & 100 \\
\hline G2 & 0.527 & 2.17 & 0.599 & 0.0821 & 3.38 & 15.6 & 64.3 & 17.7 & 2.43 & 100 \\
\hline G3 & 0.579 & 2.60 & 1.33 & 0.0777 & 4.58 & 12.6 & 56.7 & 29.0 & 1.70 & 100 \\
\hline G4 & 0.707 & 3.48 & 0.699 & 0.0738 & 4.96 & 14.3 & 70.2 & 14.1 & 1.49 & 100 \\
\hline G5 & 0.355 & 3.26 & 0.888 & 0.0612 & 4.56 & 7.79 & 71.4 & 19.5 & 1.34 & 100 \\
\hline G6 & 0.753 & 2.02 & 1.19 & 0.0682 & 4.03 & 18.7 & 50.2 & 29.5 & 1.69 & 100 \\
\hline G7 & 0.679 & 12.0 & 1.30 & 0.210 & 14.2 & 4.78 & 84.6 & 9.13 & 1.48 & 100 \\
\hline G8 & 0.950 & 11.8 & 1.64 & $<$ LOD & 14.4 & 6.61 & 82.0 & 11.4 & 0 & 100 \\
\hline G9 & 0.905 & 4.95 & 1.24 & 0.0663 & 7.16 & 12.6 & 69.1 & 17.3 & 0.926 & 100 \\
\hline G10 & 1.27 & 3.36 & 0.0905 & 0.159 & 4.88 & 26.0 & 68.9 & 1.85 & 3.25 & 100 \\
\hline
\end{tabular}


Table 4. (Continued)

\begin{tabular}{lcccccccccc}
\hline e - Copper & \multicolumn{9}{c}{$\mu \mathrm{g} / \mathrm{g}$} \\
\cline { 2 - 11 } & step 1 & step 2 & step 3 & step 4 & sum extracted & step 1 & step 2 & step 3 & step 4 & sum extracted \\
\hline G1 & LOD & 3.63 & 0.408 & 8.16 & 12.2 & 0 & 29.8 & 3.35 & 66.9 & 100 \\
G2 & <OD & 4.67 & 5.99 & 7.57 & 18.2 & 0 & 25.6 & 32.9 & 41.5 & 100 \\
G3 & LOD & 3.30 & 9.40 & 6.48 & 19.2 & 0 & 17.2 & 49.0 & 33.8 & 100 \\
G4 & < LOD & 6.14 & 0.101 & 4.00 & 10.2 & 0 & 59.9 & 0.991 & 39.1 & 100 \\
G5 & <OD & 1.72 & 15.1 & 9.48 & 24.8 & 0 & 6.90 & 60.9 & 38.2 & 100 \\
G6 & LOD & 4.00 & $<$ LOD & 4.00 & 8.00 & 0 & 50.0 & 0 & 50.0 & 100 \\
G7 & $<$ LOD & 7.43 & $<$ LOD & 4.80 & 12.2 & 0 & 60.7 & 0 & 39.3 & 100 \\
G8 & 2.94 & 7.93 & 0.307 & 6.52 & 14.8 & 19.9 & 53.7 & 2.08 & 44.2 & 100 \\
G9 & $<$ LOD & 4.94 & 0.103 & 2.49 & 7.53 & 0 & 65.6 & 2.00 & 33.1 & 100 \\
G10 & 3.10 & 66.1 & 34.1 & 56.1 & 156 & 1.98 & 42.3 & 3.00 & 35.9 & 100 \\
\hline
\end{tabular}

\begin{tabular}{lcccccccccc}
\hline f - Iron & \multicolumn{1}{c}{$\mu \mathrm{g} / \mathrm{g}$} \\
\cline { 2 - 11 } & step 1 & step 2 & step 3 & step 4 & sum extracted & step 1 & step 2 & step 3 & step 4 & sum extracted \\
\hline G1 & 5.45 & 3073 & 320 & 25490 & 28889 & 0.0189 & 10.6 & 1.11 & 88.2 & 100 \\
G2 & 3.51 & 2569 & 483 & 19607 & 22663 & 0.0155 & 11.3 & 2.13 & 86.5 & 100 \\
G3 & 5.23 & 4010 & 1200 & 18067 & 23282 & 0.0225 & 17.2 & 5.16 & 77.6 & 100 \\
G4 & 5.26 & 2252 & 590 & 28976 & 31823 & 0.0165 & 7.08 & 1.85 & 91.1 & 100 \\
G5 & 2.17 & 2587 & 527 & 17365 & 20481 & 0.0106 & 12.6 & 2.57 & 84.8 & 100 \\
G6 & 4.11 & 2594 & 341 & 25939 & 28878 & 0.0142 & 8.98 & 1.18 & 89.8 & 100 \\
G7 & 1.62 & 3053 & 942 & 28418 & 32415 & 0.00501 & 9.42 & 2.90 & 87.7 & 100 \\
G8 & 2.42 & 2987 & 511 & 33040 & 36540 & 0.00661 & 8.17 & 1.40 & 90.4 & 100 \\
G9 & 5.75 & 4201 & 1048 & 20888 & 26143 & 0.0220 & 16.1 & 4.01 & 79.9 & 100 \\
G10 & 0.922 & 1147 & 260 & 14822 & 16230 & 0.00568 & 7.07 & 1.60 & 91.3 & 100 \\
\hline
\end{tabular}

\begin{tabular}{lcccccccccc}
\hline g - Lead & \multicolumn{1}{c}{$\mu \mathrm{g} / \mathrm{g}$} \\
\cline { 2 - 11 } & step 1 & step 2 & step 3 & step 4 & sum extracted & step 1 & step 2 & step 3 & step 4 & sum extracted \\
\hline G1 & $<$ LOD & 41.7 & $<$ LOD & 7.83 & 49.5 & 0 & 84.2 & 0 & 15.8 & 100 \\
G2 & $<$ LOD & 39.0 & $<$ LOD & 6.57 & 45.6 & 0 & 85.6 & 0 & 14.4 & 100 \\
G3 & $<$ LOD & 40.9 & $<$ LOD & 7.09 & 48.0 & 0 & 85.2 & 0 & 14.8 & 100 \\
G4 & $<$ LOD & 19.9 & $<$ LOD & 7.83 & 27.8 & 0 & 71.8 & 0 & 28.2 & 100 \\
G5 & $<$ LOD & 29.1 & $<$ LOD & 9.49 & 38.6 & 0 & 75.4 & 0 & 24.6 & 100 \\
G6 & $<$ LOD & 24.9 & $<$ LOD & 3.92 & 28.8 & 0 & 86.4 & 0 & 13.6 & 100 \\
G7 & $<$ LOD & 36.5 & $<$ LOD & 9.64 & 46.2 & 0 & 79.1 & 0 & 20.9 & 100 \\
G8 & $<$ LOD & 34.9 & $<$ LOD & 13.2 & 48.1 & 0 & 72.5 & 0 & 27.5 & 100 \\
G9 & $<$ LOD & 15.5 & $<$ LOD & 5.11 & 20.6 & 0 & 75.2 & 0 & 24.8 & 100 \\
G10 & 0.503 & 11.3 & $<$ LOD & 4.34 & 16.1 & 3.12 & 70.0 & 0 & 26.9 & 100 \\
\hline
\end{tabular}

\begin{tabular}{lcccccccccc}
\hline h - Magnesium & \multicolumn{1}{c}{$\mu \mathrm{g} / \mathrm{g}$} \\
\cline { 2 - 11 } & step 1 & step 2 & step 3 & step 4 & sum extracted & step 1 & step 2 & step 3 & step 4 & sum extracted \\
\hline G1 & 3382 & 4832 & 597 & 5340 & 14150 & 23.9 & 34.1 & 4.22 & 37.7 & 100 \\
G2 & 3554 & 5329 & 440 & 3818 & 13142 & 27.0 & 40.5 & 3.35 & 29.1 & 100 \\
G3 & 3747 & 4449 & 729 & 3700 & 12624 & 29.7 & 35.2 & 5.77 & 29.3 & 100 \\
G4 & 499 & 398 & 324 & 3226 & 4446 & 11.2 & 8.94 & 7.28 & 72.6 & 100 \\
G5 & 3364 & 6485 & 2149 & 2451 & 14449 & 23.3 & 44.9 & 14.9 & 17.0 & 100 \\
G6 & 2400 & 2956 & 172 & 4227 & 9754 & 24.6 & 30.3 & 1.76 & 43.3 & 100 \\
G7 & 499 & 398 & 324 & 3226 & 4446 & 11.2 & 8.94 & 7.28 & 72.6 & 100 \\
G8 & 2193 & 1089 & 318 & 2861 & 6461 & 33.9 & 16.9 & 4.92 & 44.3 & 100 \\
G9 & 320 & 1285 & 385 & 2999 & 4990 & 6.40 & 25.8 & 7.71 & 60.1 & 100 \\
G10 & 6731 & 6916 & 1068 & 2537 & 17252 & 39.0 & 40.1 & 6.19 & 14.7 & 100 \\
\hline
\end{tabular}


Table 4. (Continued)

\begin{tabular}{lcccccccccc}
\hline i - Manganese & \multicolumn{1}{c}{$\mu \mathrm{g} / \mathrm{g}$} \\
\cline { 2 - 11 } & step 1 & step 2 & step 3 & step 4 & sum extracted & step 1 & step 2 & step 3 & step 4 & sum extracted \\
\hline G1 & 88.2 & 191 & 6.84 & 113 & 400 & 22.0 & 47.9 & 1.71 & 28.4 & 100 \\
G2 & 84.4 & 123 & 3.79 & 105 & 316 & 26.7 & 38.9 & 1.20 & 33.3 & 100 \\
G3 & 89.5 & 143 & 7.36 & 89.3 & 329 & 27.2 & 43.4 & 2.24 & 27.2 & 100 \\
G4 & 84.7 & 172 & 5.07 & 107 & 369 & 23.0 & 46.7 & 1.38 & 28.9 & 100 \\
G5 & 76.7 & 204 & 6.81 & 82.9 & 370 & 20.7 & 55.0 & 1.84 & 22.4 & 100 \\
G6 & 65.6 & 70.6 & 2.77 & 88.8 & 228 & 28.8 & 31.0 & 1.22 & 39.0 & 100 \\
G7 & 78.3 & 305 & 8.01 & 186 & 577 & 13.6 & 52.8 & 1.39 & 32.2 & 100 \\
G8 & 91.3 & 324 & 8.80 & 213 & 637 & 14.3 & 50.8 & 1.38 & 33.5 & 100 \\
G9 & 113 & 436 & 19.8 & 132 & 700 & 16.1 & 62.2 & 2.83 & 18.8 & 100 \\
G10 & 54.9 & 152 & 11.8 & 134 & 353 & 15.6 & 43.2 & 3.35 & 37.9 & 100 \\
\hline
\end{tabular}

\begin{tabular}{lcccccccccc}
\hline j - Nickel & \multicolumn{1}{c}{$\mu \mathrm{g} / \mathrm{g}$} \\
\cline { 2 - 11 } & step 1 & step 2 & step 3 & step 4 & sum extracted & step 1 & step 2 & step 3 & step 4 & sum extracted \\
\hline G1 & 1.11 & 5.08 & 3.39 & 25.4 & 34.9 & 3.18 & 14.5 & 9.70 & 72.6 & 100 \\
G2 & 1.01 & 3.56 & 4.29 & 15.6 & 24.4 & 4.15 & 14.6 & 17.6 & 63.7 & 100 \\
G3 & 1.13 & 4.54 & 6.03 & 14.5 & 26.2 & 4.33 & 17.3 & 23.0 & 55.3 & 100 \\
G4 & 0.786 & 2.84 & 4.29 & 19.1 & 27.1 & 2.91 & 10.5 & 15.9 & 70.7 & 100 \\
G5 & 1.12 & 3.82 & 3.65 & 15.4 & 24.0 & 4.66 & 15.9 & 15.2 & 64.3 & 100 \\
G6 & 0.721 & 3.21 & 3.67 & 22.0 & 29.6 & 2.44 & 10.9 & 12.4 & 74.3 & 100 \\
G7 & 2.48 & 10.7 & 7.09 & 30.2 & 50.5 & 4.90 & 21.2 & 14.0 & 59.9 & 100 \\
G8 & 3.59 & 18.4 & 8.60 & 69.2 & 99.7 & 3.60 & 18.4 & 8.62 & 69.4 & 100 \\
G9 & 1.74 & 2.00 & 5.99 & 16.4 & 26.1 & 6.64 & 7.65 & 22.9 & 62.8 & 100 \\
G10 & 2.67 & 5.63 & 1.99 & 34.9 & 45.1 & 5.91 & 12.5 & 4.41 & 77.2 & 100 \\
\hline
\end{tabular}

\begin{tabular}{lcccccccccc}
\hline k - Potassium & \multicolumn{1}{c}{$\mu \mathrm{g} / \mathrm{g}$} \\
\cline { 2 - 11 } & step 1 & step 2 & step 3 & step 4 & sum extracted & step 1 & step 2 & step 3 & step 4 & sum extracted \\
\hline G1 & 1039 & 310 & $<$ LOD & 1410 & 2759 & 37.7 & 11.2 & 0 & 51.1 & 100 \\
G2 & 685 & 261 & 52.0 & 1078 & 2076 & 33.0 & 12.6 & 2.51 & 51.9 & 100 \\
G3 & 607 & 261 & 82.0 & 1058 & 2008 & 30.2 & 13.0 & 4.08 & 52.7 & 100 \\
G4 & 605 & 272 & $<$ LOD & 690 & 1567 & 38.6 & 17.4 & 0.00 & 44.1 & 100 \\
G5 & 297 & 135 & 217 & 443 & 1091 & 27.2 & 12.4 & 19.9 & 40.5 & 100 \\
G6 & 301 & 153 & $<$ LOD & 1010 & 1464 & 20.6 & 10.4 & 0 & 69.0 & 100 \\
G7 & 171 & 90.0 & $<$ LOD & 1010 & 1271 & 13.5 & 7.08 & 0 & 79.5 & 100 \\
G8 & 280 & 115 & $<$ LOD & 725 & 1120 & 25.0 & 10.2 & 0 & 64.7 & 100 \\
G9 & 178 & 118 & $<$ LOD & 575 & 871 & 20.4 & 13.6 & 0 & 66.0 & 100 \\
G10 & 542 & 320 & 46.2 & 1562 & 2470 & 22.0 & 12.9 & 1.87 & 63.2 & 100 \\
\hline
\end{tabular}

\begin{tabular}{lcccccccccc}
\hline 1 - Zinc & \multicolumn{9}{c}{$\mu \mathrm{g} / \mathrm{g}$} \\
\cline { 2 - 11 } & step 1 & step 2 & step 3 & step 4 & sum extracted & step 1 & step 2 & step 3 & step 4 & sum extracted \\
\hline G1 & 16.9 & 50.9 & $<$ LOD & 72.0 & 140 & 12.1 & 36.4 & 0 & 51.5 & 100 \\
G2 & 16.9 & 42.8 & 4.69 & 47.7 & 112 & 15.1 & 38.2 & 4.19 & 42.5 & 100 \\
G3 & 25.4 & 56.8 & 7.26 & 47.2 & 137 & 18.6 & 41.6 & 5.31 & 34.6 & 100 \\
G4 & 2.87 & 16.9 & 0.304 & 50.2 & 70.3 & 4.07 & 24.1 & 0.432 & 71.4 & 100 \\
G5 & 12.0 & 42.2 & 45.0 & 46.0 & 145 & 8.26 & 29.1 & 31.0 & 31.7 & 100 \\
G6 & 7.16 & 26.5 & $<$ LOD & 57.9 & 91.6 & 7.82 & 29.0 & 0 & 63.2 & 100 \\
G7 & 8.79 & 32.3 & 6.49 & 47.3 & 94.8 & 9.27 & 34.0 & 6.84 & 49.9 & 100 \\
G8 & 6.36 & 42.7 & $<$ LOD & 65.1 & 114 & 5.57 & 37.4 & 0 & 57.0 & 100 \\
G9 & 0.149 & 5.91 & 1.14 & 31.5 & 38.7 & 0.385 & 15.3 & 2.94 & 81.4 & 100 \\
G10 & $<$ LOD & 5.31 & $<$ LOD & 61.1 & 66.4 & 0 & 7.99 & 0 & 92.0 & 100 \\
\hline
\end{tabular}


ranged from 1.2 up to $2.1 \mu \mathrm{g} / \mathrm{g}$ and correspond to values in literature: $\mathrm{Cd}$ levels were found in the Yozgat region (Turkey) below $1 \mu \mathrm{g} / \mathrm{g},{ }^{16}$ in the Incesu-Kayseri region (Turkey) $1-4 \mu \mathrm{g} / \mathrm{g},{ }^{17}$ from $<0.1 \mu \mathrm{g} / \mathrm{g}$ to $1.5 \mu \mathrm{g} / \mathrm{g}$ for horticultural soils in New Zealand, ${ }^{18}$ a study on agricultural soils in USA led to $\mathrm{Cd}$ concentrations of $<0.01$ $\mu \mathrm{g} / \mathrm{g}$ to $2.0 \mu \mathrm{g} / \mathrm{g}$. ${ }^{19}$ In orchard soils in China total $\mathrm{Cd}$ amounts of 0.7 up to $1.8 \mu \mathrm{g} / \mathrm{g}$ were found by Li et al. ${ }^{20}$ In Algeria in a study on agricultural soils $\mathrm{Cd}$ level of $0.30 \mu \mathrm{g} / \mathrm{g}$ (median) was obtained. ${ }^{21}$ Cadmium levels in horticultural and agricultural soils depend on the continued use of phosphate-fertilizers and zinc containing agrichemicals, where cadmium is present as impurity. ${ }^{15}$ The total amounts of cadmium in garden soils were expected to be lower than those reported for plants grown commercially. Cadmium contamination limits agricultural use of soil, thus critical soil Cd concentration has been proposed in various countries, e.g. $0.3 \mu \mathrm{g} / \mathrm{g}$ in China. ${ }^{3}$ According to French regulation maximum Cd soil level is $2 \mu \mathrm{g} / \mathrm{g} .{ }^{14}$ In Canada the limit concentration is $1.4 \mu \mathrm{g} / \mathrm{g}^{2}$

Regarding the extractability of cadmium, 30-60\% were extracted in the first two steps $(10-20 \%$ and 20 $40 \%$, resp.) and the remaining 40-70\% were found in the residue (step 4).

\section{Chromium}

Chromium is ubiquitous in the environment, like soil, water and air, and occurs in two forms, as Cr(III) and $\mathrm{Cr}(\mathrm{VI})$. The latter is more toxic and, in plants affects negatively leaf and root growth, inhibits certain enzymes and may cause mutagenesis. ${ }^{22}$ High chromium levels in soil are caused by anthropomorphic activities. The amounts found in the analysed soils samples ranged from 10 up to $63 \mu \mathrm{g} / \mathrm{g}$ with $70 \%$ between 19 and $30 \mu \mathrm{g} / \mathrm{g}$. The exceptions are G6 $11 \mu \mathrm{g} / \mathrm{g}, \mathrm{G} 741 \mu \mathrm{g} / \mathrm{g}$ and G8 $63 \mu \mathrm{g} / \mathrm{g}$. The entire range is reported by Adriano $^{4}$ as normal chromium soil level, namely 10 to $50 \mu \mathrm{g} / \mathrm{g}$ depending on the bedrock concentration. In Algeria (Anaba region) the median total chromium level in agricultural soil was found to be $28.3 \mu \mathrm{g} / \mathrm{g}$ (Ref. 21) and in the Incesu-Kayseri region (Turkey) 0.5-38 $\mu \mathrm{g} / \mathrm{g} .{ }^{17}$ Even the outlier G8 is below the permissible concentration of toxic elements for agricultural soils of China $(200 \mu \mathrm{g} / \mathrm{g}){ }^{3}$ French regulatory limit of $150 \mu \mathrm{g} / \mathrm{g}$ (Ref. 14) and the Canadian limit of $64 \mu \mathrm{g} / \mathrm{g}$ for total chromium. ${ }^{2}$ Regarding the extractability of chromium from soil it was found that $1 \%$ was extracted in step 1 and up to $10 \%$ in steps 2 and 3 . As expected, the highest amounts were found in samples G7 and G8.

\section{Cobalt}

The French regulations' limit of cobalt in soil is $30 \mu \mathrm{g} / \mathrm{g}$ (Ref. 14) and in Canada $40 \mu \mathrm{g} / \mathrm{g}^{2}$ All soils analysed contain $\mathrm{Co}$ in lower concentration. In the investigated soil samples six of ten had cobalt levels between 4 and 5 $\mu \mathrm{g} / \mathrm{g}$. Higher levels were found in G9 $(7.2 \mu \mathrm{g} / \mathrm{g})$ and in G7 and G8 (approx. $14 \mu \mathrm{g} / \mathrm{g}$ ). G2 has less Co, namely $3.4 \mu \mathrm{g} / \mathrm{g}$. Three Turkish studies came to similar results. In the soil samples from Kayseri region the concentration of cobalt is found to be less than $3 \mu \mathrm{g} / \mathrm{g},{ }^{23}$ in the Incesu-Kayseri region $0.5-38 \mu \mathrm{g} / \mathrm{g}$ (Ref. 17) and in the Yozgat region $4-8 \mu \mathrm{g} / \mathrm{g} .{ }^{16}$ The China National Environmental Monitoring Centre reported cobalt levels of $15 \mu \mathrm{g} / \mathrm{g}$ and $26 \mu \mathrm{g} / \mathrm{g}$. ${ }^{24}$ In plants cobalt is required by the urease enzyme. Cobalt has only a low affinity to humic substances, ${ }^{12}$ and it is adsorbed to clay minerals in soil and geological origin by specific reactions. ${ }^{25}$ This is reflected in its extraction behaviour: $50-70 \%$ of Co is extracted in step 2, approx. $10 \%$ in step 1 and up to 30 $\%$ in step 3 . Only $3 \%$ of the total cobalt were detected in the residue (step 4).

\section{Copper}

Copper is essential for plants but is toxic at higher levels. The total copper levels in the soil samples analysed ranged from 10 to $25 \mu \mathrm{g} / \mathrm{g}$, except for G10 containing $156 \mu \mathrm{g} / \mathrm{g}$. Similar levels were reported for Turkish soil samples, namely $12-27.5 \mu \mathrm{g} / \mathrm{g}$ from Kayseri region ${ }^{23}$ and ranging from 16-26 $\mu \mathrm{g} / \mathrm{g}$ in Incesu-Kayseri region, ${ }^{7}$ and even slightly higher than in samples from the Yozgat region $(4-14 \mu \mathrm{g} / \mathrm{g}),{ }^{16}$ and below those reported for agricultural soils $(40 \mu \mathrm{g} / \mathrm{g}$ in market garden and $58 \mu \mathrm{g} / \mathrm{g}$ orchard), ${ }^{18}$ what can be explained by the minor use of copper-based fungicides in private gardens than in horticulture. In agricultural soils in the USA levels from 0.3 up to $495 \mu \mathrm{g} / \mathrm{g}$ were found with a median of $18.5 \mu \mathrm{g} / \mathrm{g} .{ }^{19}$ Algerian agricultural soils had a median copper level of $23.8 \mu \mathrm{g} / \mathrm{g},{ }^{21}$ while higher copper levels were found in Italian vineyard soils $(945 \mu \mathrm{g} / \mathrm{g}) .{ }^{26} \mathrm{Chi}-$ nese and French regulations stipulate maximum copper level in soil of $100 \mu \mathrm{g} / \mathrm{g}$. ${ }^{3,14}$ The Canadian Ministry of Environment limits $\mathrm{Cu}$ in agricultural soil to $63 \mu \mathrm{g} / \mathrm{g}{ }^{2}$ Copper plays a role in plants' growth. Common garden plants, like tomatoes and strawberries need $\mathrm{Cu}$ up to 20 $\mu \mathrm{g} / \mathrm{g}$, thus all soils of this study met these needs. ${ }^{5}$

Regarding the extractability it was found that copper was leached mainly from soil in step 2 (reducible fraction). A similar finding was reported by Whalley and Grant. ${ }^{27}$ In step 1, only extracts of G10 was the copper level above the LOD, which could be a result of the high total concentration in the sample. $\mathrm{Cu}$ has a high affinity for humic substances. ${ }^{12}$

\section{Lead}

The total lead content found ranged from $16-50 \mu \mathrm{g} / \mathrm{g}$. A wide range of its concentrations are reported for soils in New Zealand, ${ }^{15}$ namely $11-251 \mu \mathrm{g} / \mathrm{g}$ and in the Kayseri region (Turkey) 16-83 $\mu \mathrm{g} / \mathrm{g}$ (Ref. 23) and in the IncesuKayseri region (Turkey) $13-34 \mu \mathrm{g} / \mathrm{g} .{ }^{16}$ Elevated lead levels are attributed to the usage of lead arsenate as insecticides especially in orchards. Similar levels were 
found for American agricultural soils, namely $<1-135$ $\mu \mathrm{g} / \mathrm{g} .{ }^{19}$ In Algeria (Anaba region) lead levels of approx. $40 \mu \mathrm{g} / \mathrm{g}$ were found, ${ }^{21}$ whereby the highest values were found in soils of the city centre. Due to lead-free gasoline the lead concentration in soil decreased during the last decades. Levels up to $720 \mu \mathrm{g} / \mathrm{g}$ were found in soils in the USA. ${ }^{28}$ At present lead originates as contaminant of certain fertilisers ans sewage sludge. French regulations limit lead levels in soil at $100 \mu \mathrm{g} / \mathrm{g} .{ }^{14}$ The Canadian Ministry of Environment ${ }^{2}$ limits in agricultural soils the lead level to a maximum of $70 \mu \mathrm{g} / \mathrm{g}$. In China the limit is $300 \mu \mathrm{g} / \mathrm{g}^{3}$

Lead is mainly extracted in steps 2 and 4 while in the solutions after step 1 and 3 the lead concentrations were below the LOD. Exception is G10 having the lowest lead content, but with $3 \%$ extracted in step $1 . \mathrm{Pb}$ is readily complexed by humic substances. ${ }^{12}$

\section{Manganese}

In soils from Algeria Maas and colleagues ${ }^{21}$ found Mn content ranging from 34 to $636 \mu \mathrm{g} / \mathrm{g}$, the median being $400 \mu \mathrm{g} / \mathrm{g}$. Similar values were found in Turkey, namely, in the Incesu-Kayseri region $130-2600 \mu \mathrm{g} / \mathrm{g},{ }^{16}$ in the Yozgat region $280-360 \mu \mathrm{g} / \mathrm{g},{ }^{17}$ and in the Kayseri region $500 \mu \mathrm{g} / \mathrm{g} .^{23}$ The last two findings were in roadside soils. In both cases there was no correlation found between Mn content in soil and distance to the road. Manganese is not easily bound to humic substances. ${ }^{12}$ The total manganese contents in the analysed garden soils ranged from 300 to $400 \mu \mathrm{g} / \mathrm{g}$. Less $\mathrm{Mn}$ was found in G6 $(228 \mu \mathrm{g} / \mathrm{g})$ and 600 to $700 \mu \mathrm{g} / \mathrm{g}$ in G7-G9. No regulations exist for manganese content in agricultural soils. ${ }^{2,14}$ Manganese is involved carbohydrate and nitrogen metabolism, but deficiency is not likely to occur in private gardens. Levels up to $500 \mu \mathrm{g} / \mathrm{g}$ are needed by some garden plants. ${ }^{5}$

Most manganese is extracted in step 2, 30 up to 50 $\%$. In G9, $60 \%$ were extracted and 20 to $40 \%$ were found in the residue (step 4). In the oxidisable fraction only up to $3 \%$ of the total Mn amount were found.

Nickel

For nickel similar extraction behaviour was reported in literature $^{27}$ with the highest extraction yields in the first two steps. The soils analysed in the present were different, since less than $10 \%$ were extracted in step 1 , and up to $20 \%$ in step 2, while the rest remained unextracted. Not only can the different soil composition determined by the parent rock be considered as reason for this, but also in the diverse experimental set up. Whereas Whalley and colleague ${ }^{27}$ first let the metals adsorb to the soil, real soil samples were used in the present investigation. The nickel content found in the present study ranged from 24 up to $35 \mu \mathrm{g} / \mathrm{g}$, except for G10 $(45 \mu \mathrm{g} / \mathrm{g}), \mathrm{G} 7(51 \mu \mathrm{g} / \mathrm{g})$ and G8 $(100 \mu \mathrm{g} / \mathrm{g})$. Holmgren et al. ${ }^{19}$ reported a soil in a similar range, namely with Nickel from 0.7 up to $269 \mu \mathrm{g} / \mathrm{g}$, with a median of
$18.2 \mu \mathrm{g} / \mathrm{g}$. They found higher Ni levels in serpentine soils and in glaciated areas. Nickel amount in to the soil samples from Kayseri region (Turkey) is $43-82 \mu \mathrm{g} / \mathrm{g},{ }^{23}$ in the Incesu-Kayseri region (Turkey) $12-41 \mu \mathrm{g} / \mathrm{g}$ (Ref. 16) and in the Yozgat region (Turkey) $8-29 \mu \mathrm{g} / \mathrm{g},{ }^{17}$ in all cases similar to the obtained results. According to French regulations the allowed maximum $\mathrm{Ni}$ soil level is $50 \mu \mathrm{g} / \mathrm{g} .{ }^{14}$ The same limit value is applied to Chinese agricultural soils. ${ }^{3}$

Zinc

Agrochemicals, such as fungicides and fertilisers, are a potential source of zinc in agriculture. Zinc levels in soils ranged from $64-108 \mu \mathrm{g} / \mathrm{g}$ in New Zealand, ${ }^{15}$ in the Kayseri region (Turkey) from $15-25 \mu \mathrm{g} / \mathrm{g},{ }^{23}$ in the Incesu-Kayseri region (Turkey) from $26-51 \mu \mathrm{g} / \mathrm{g},{ }^{16}$ in the Lower Fraser Valley of British Columbia from 43-107 $\mu \mathrm{g} / \mathrm{g},{ }^{29}$ and in rural soils in Netherlands from 7-95 $\mu \mathrm{g} / \mathrm{g} .{ }^{30}$ The results of the present study are in the same order of magnitude, namely from $40-145 \mu \mathrm{g} / \mathrm{g}$. Zinc levels up to $560 \mu \mathrm{g} / \mathrm{g}$ (median $65 \mu \mathrm{g} / \mathrm{g}$ ) were found in Algeria. ${ }^{21}$ Regulations limit $\mathrm{Zn}$ levels in soil to $300 \mu \mathrm{g} / \mathrm{g}$ in France ${ }^{14}$ and to $250 \mu \mathrm{g} / \mathrm{g}$ in China. ${ }^{3}$ Zinc is involved in carbohydrate metabolism and in enzymatic systems regulating plant growth. Up to $100 \mu \mathrm{g} / \mathrm{g}$ zinc is needed by tomatoes, ${ }^{5}$ and $5-30 \mu \mathrm{g} / \mathrm{g}$ by strawberries, blueberries and grapes.

Zinc is leached mainly in step 2 while small amounts were extracted in steps 1 and 3 .

\section{CONCLUSIONS}

All soils analysed contained the studied elements at levels reported for other geographical regions worldwide and the levels did not exceed the limits imposed by different jurisdictions. The three soil samples taken from the city of Zagreb contained higher levels of pollutants. A similar observation was made in twelve cities in China. ${ }^{31}$ The nutrient supply of elements was found to be adequate in all tested soil samples.

\section{REFERENCES}

1. R. E. Pugh, D. G. Dick, and A. L. Fredeen, Ecotox. Environ. Safe. 52 (2002) 273-279.

2. Canadian Council of Ministers of the Environment. 2012. Soil Quality Guidelines for the Protection of Environmental and $\mathrm{Hu}-$ man Health. Accessed 4 June 2013 from http://st-ts.ccme.ca/.

3. CEPA (Chinese Environmental Protection Administration, Environmental Quality Standard for Soils (GB15618-1995), Beijing, 1995.

4. D. C. Adriano, Trace Elements in Terrestrial Environments: Biogeochemistry, Bioavailability, and Risks of Metals, $2^{\text {nd }}$ edition, Springer, New York, 2001.

5. C. O. Plank and D. E. Kissel, Plant Analysis Handbook for Georgia, online version. Accessed 4 June 2013, from http://aesl.ces.uga.edu/publications/plant/index.htm. 
6. A. Łozak, K. Sołtyk, P. Ostapczuk, and Z. Fijałek, Sci. Tot. Environ. 289 (2002) 33-40.

7. C. R. M. Rao, A. Sahuquillo, and J. F. Lopez Sanchez, Water Air Soil Poll. 189 (2008) 291-333.

8. A. M. Ure, Ph. Quevauviller, H. Muntau, and B. Griepink, Int. J. Environ. Anal. Chem. 51 (1993) 135-151.

9. J. F. López-Sánchez, A. Sahuquillo, G. Rauret, M. Lachica, E. Barahona, A. Gomez, A. M. Ure, H. Muntau, and Ph. Quevauviller, Extraction procedures for soil analysis, in: $\mathrm{Ph}$. Quevauviller (Ed.), Methodologies in soil and sediment fractionation studies, Publisher Royal Society of Chemistry, Cambridge, 2002, pp. 28-65.

10. M. Zeiner, I. Juranović Cindrić, I. Lovrenčić Mikelić, G. Medunić, Š. Kampić, N. Tomašić, and G. Stingeder, Environ. Monit. Assess. 185 (2013) 223-229.

11. B. Marin, M. Valladon, and M. A. Polve, Anal. Chim. Acta 342 (1997) 91-112.

12. W. G. Botero, L. Camargo de Oliveira, J. C. Rocha, A. H. Rosa, and A. dos Santos, J. Haz. Mat. 177 (2010) 307-311.

13. X. Li and I. Thornton, App. Geochem. 16 (2001) 1693-1706.

14. Agence Française de NORmalisation (AFNOR), 1996. Qualité des sols. Recueil de normes Françaises 1996, AFNOR, Paris.

15. WHO, 1992. Cadmium. In: Environmental Health Criteria, vol. 134. WHO, Geneva.

16. M. Soylak, Y. Akkaya, L. Elci, Trace Elements and Electrolytes 18 (2001) 176-180.

17. Y .E. Unsal, E. Yilmaz, M. Soylak, M. Tuzen, Fresenius Environmental Bulletin 22 (2013) 1441-1445.
18. S. K. Gaw, A. L. Wilkins, N. D. Kim, G. T. Palmer, and P. Robinson, Sci. Tot. Environ. 355 (2006) 31- 47.

19. G. G. S. Holmgren, M. W. Meyer, R. L. Chaney, and R. B. Daniels, J. Environ. Qual. 22 (1993) 335-348.

20. J. T. Li, J. W. Qiu, X. W. Wang, Y. Zhong, C. Y. Lan, and W. S. Shu, Environ. Poll. 143 (2006) 159-165.

21. S. Maas, R. Scheifler, M. Benslama, N. Crini, E. Lucot, Z. Brahmia, S. Benyacoub, and P. Giraudoux, Environ. Poll. 158 (2010) 2294-2301.

22. J. L. Gardea-Torresdey, J. R. Peralta-Videa, M. Montes, G. de la Rosa, and B. Corral-Diaz, Biores. Technol. 92 (2004) 229-235.

23. M. Soylak, O. Türkoglu, Journal of Trace and Microprobe Techniques 17 (1999) 209-217.

24. G. Jin, Y. Zhu, W. Jiang, B. Xie, and B. Cheng, Analyst 122 (1997) 263-265.

25. K. G. Tiller, J. F. Hodgoson, and M. Peech, Soil Sci. 95 (1963) 392-399.

26. A. Deluisa, P. Giandon, M. Aichner, P. Bortolami, L. Bruna, A. Lupetti, F. Nardelli, and G. Stringari, Comm. Soil Sci. Plant Anal. 27 (1996) 1537-1548.

27. C. Whalley and A. Grant, Anal. Chim. Acta 291 (1994) 287-295.

28. I. Merwin, P.T. Pruyne, J. G. Ebel, K. L. Manzell, and D. J. Lisk, Chemosphere 29 (1994) 1361-1367.

29. L. A. De Pieri, W. T. Buckley, and C. G. Kowalenko, Can. J. Soil. Sci. 76 (1996) 173-182.

30. P. F. M. Van-Gaans, S. P. Vriend, S. Bleyerveld, G. Schrage, and A. Vos, Environ. Moni. Assess. 34 (1995) 73-102.

31. B. Wei and L. Yang, Microchem. J. 94 (2010) 99-107. 